\title{
Hyperglycemia Damages the Endothelial Glycocalyx: Implications for The Blood Brain Barrier
}

\author{
Sandra V Lopez-Quintero*1,2 and David C Spray ${ }^{1}$ \\ ${ }^{1}$ Department of Neuroscience, Albert Einstein College of Medicine, Rose F. Kennedy Center, USA \\ ${ }^{2}$ Department of Mechanical Engineering, Manhattan College, USA
}

*Corresponding author: Sandra Veronica Lopez-Quintero, Department of Mechanical Engineering, Manhattan College, 4513 Manhattan College Parkway, Riverdale, NY 10471, USA.

To Cite This Article: Sandra Veronica Lopez-Quintero, Hyperglycemia Damages the Endothelial Glycocalyx: Implications for The Blood Brain Barrier. Am J Biomed Sci \& Res. 2019 - 6(5). AJBSR.MS.ID.001072. DOI: 10.34297/AJBSR.2019.06.001072.

Received: 畊 December 13, 2019; Published: 監 December 17, 2019

\begin{abstract}
The glycocalyx is a matrix of proteoglycans, glycoproteins, glycosaminoglycans, glycolipids and soluble proteins that coats the endothelial cells, together with the endothelium, the astrocyte end feet and basement membrane, contributes to the blood brain barrier, which prevents large molecules, neurotoxins, inflammatory factors and pathogens into the brain. It has been demonstrated that hyperglycemia results in damage to the glycocalyx through mechanisms that are still being elucidated. This means that the brain may be exposed to harmful agents that can cause multiple diseases. More studies are therefore needed to understand and prevent brain disease due to hyperglycemia.
\end{abstract}

Keywords: Hyperglycimia; Diabetes; Brain; Blood brain barrier; Endothelium; Glycocalyx; Cerebrovasculature

\section{Introduction}

It is well established that diabetes can lead to cerebral vasodegeneration, manifested principally by blood brain barrier (BBB) dysfunction [1]. The BBB consists of a tight capillary network that protects the brain from most circulating molecules while providing access to necessary nutrients required for function. These capillaries are formed by an inner layer of endothelial cells that are joined together via tight junctions and adherent junctions followed by a less dense layer of pericytes (ratio 4:1) that form socket like structures at the point of contact with endothelial cells, and a layer of astrocytes, whose end feet are in close proximity with the endothelium and allow for the diffusion of metabolites and neurotransmitters from the brain tissue to the endothelium and vice-versa [2]. The tightness of the barrier is mainly determined by the integrity of the endothelium, which provides the major resistance to transport across the vessel walls [3].

Homeostasis of the endothelium not only depends on the adequate transmission of biochemical signals but also on dynamic interactions due to the circulating blood. The release of metabolic molecules, as well as changes in gene and protein levels have been attributed, among other stimuli, to fluid flow shear stress (FFSS)
[4]. Endothelial cells sense and respond to such stimuli due to the presence of a mechanosensing machinery that includes the glycocalyx (GCX) at the luminal side of the vessel that connects to the integrin binding domains and focal adhesions and basal GCX at the abluminal side via the cytoskeleton [5]. This mechano-signaling modulates vasculogenesis, permeability, vasoconstriction, cell death and other physiological and adaptation responses [6]. The GCX is denser in the brain, covering the entire luminal surface of cerebral capillaries, suggesting that it may be an important component of the BBB [7].

The GCX is a matrix of proteoglycans (PG), glycoproteins, glycosaminoglycans (GAGs), glycolipids and soluble proteins coating the endothelial cells. The most abundant GAGs are heparan sulfate (HS), chondroitin sulfate and hyaluronan (HA). The main PGs are syndecan (SDC)-1, -2 and -4 and glypican-1(GPC1).For reviews on the GCX composition and function see Reitsma et al. and Curry and Adamson [8,9]. The integrity of the GCX is critical for sensing FFSS caused by the circulating blood [10]. Interestingly, FFSS can modulate the GCX barrier properties against cellular infiltration and molecular transport across the vascular wall [11- 
13]. In addition, the GCX is negatively charged due to the presence of anionic oligosaccharides, thereby forming an electrical barrier $[14,15]$. In vitro experiments have shown that high glucose leads to the degradation of the HS component of the GCX in bovine aortic endothelial cells (BAEC) [16] and in human endothelial cell lines [17]. In BAEC, this resulted in an abnormal response of FFSS dependent hydraulic conductivity across the cell layer. In vivo experiments have shown that either neutral or anionic dextrans with molecular weights of $70-\mathrm{kDa}$ and higher, do not penetrate the GCX and are therefore retained in the vasculature [18], however, short-term hyperglycemia can impair the ability of the GCX to exclude 70-kDa dextran [19] possibly due to enzymatic shedding of some of its components. This was partially supported by the observation that the GCX of mice deficient in hyaluronidase 1 , an enzyme that cleaves HA, was preserved from damage in streptozotocin-induced diabetes [20]. In type 1 diabetes patients and in acute hyperglycemia, plasma HA and hyaluronidase are increased [21,22]. In type 2 male patients, sublingual and retinal GCX thickness was reduced as measured by angiography, with a concomitant increase in markers for HA catabolism (HA and hyaluronidase) and an increase in transcapillary efflux of albumin, that was partially restored following administration of sulodexide [23], a purified GAG combining HS and dermatan sulfate with affinities for antithrombin III and for heparin cofactor II respectively $[24,25]$. It is well established that reactive oxygen species (ROS) and advanced glycation end products (AGE) are involved in diabetic complications. ROS can depolymerize GAGs [26] and AGE degrade and inhibit the production of HA [27]. Besides the presence of ROS and AGE, the activation of heparanase and hyaluronidase lead to the deterioration of the GCX during diabetes [28]. The GCX therefore has been suggested as a target in diabetic vascular complications [29]. In hyperglycemic conditions, heparin or insulin preserves GAGs; insulin is an effective diabetic therapy since it both lowers blood glucose and protects the endothelium [30].

The GCX, the endothelium, the astrocyte end feet and basement membrane contribute to the BBB [31] preventing large molecules, neurotoxins, inflammatory factors and pathogens into the brain. A compromise in the BBB precedes many neurological disorders and is related to diabetes-associated comorbidities such as cognitive impairment and depression [32]. Hyper and hypoglycemia have been associated with oxidative stress in the brain, that may upregulate and activate the receptor for AGE, which also transports amyloid-beta from the blood into the brain, thus, establishing a relationship between type 2 diabetes mellitus and Alzheimer's disease (also referred to as "type 3 diabetes"). Degradation of the GCX at the BBB may imply that there is a lower FFSS-induced endothelial nitric oxide production [33] which can impair vasodilation. According to the International Diabetes Federation (www.idf.org), in 2019, approximately 463 million adults were living with diabetes and it is estimated that by 2045 this number will rise to 700 million. Therefore, more research is necessary to understand the mechanisms by which hyperglycemia damages the GCX. Such studies will likely provide a key to prevent complications in micro and macrovascular beds of the brain and other organs.

\section{Acknowledgements}

We would like to thank the National Institute of Health for their funding of project 5R01NS092466-07.

\section{Conflict of Interest}

We would like to declare that no conflict of interest exists related to this article.

\section{References}

1. Huber JD (2008) Diabetes, cognitive function, and the blood-brain barrier. Curr Pharm Des 14(16): 1594-1600.

2. Abbott NJ (2002) Astrocyte-endothelial interactions and blood-brain barrier permeability. J Anat 200(6): 629-638.

3. Abbott NJ, Patabendige AA, Dolman DE, Yusof SR, Begley DJ (2010) Structure and function of the blood-brain barrier. Neurobiol Dis 37(1): 13-25.

4. Li YS, Haga JH, Chien S (2005) Molecular basis of the effects of shear stress on vascular endothelial cells. J Biomech 38(10): 1949-1971.

5. Zeng Y, Zhang XF, Fu BM, Tarbell JM (2018) The Role of Endothelial Surface Glycocalyx in Mechanosensing and Transduction. Adv Exp Med Biol 1097: 1-27.

6. Deng Q, Huo Y, Luo J (2014) Endothelial mechanosensors: the gatekeepers of vascular homeostasis and adaptation under mechanical stress. Sci China Life Sci 57(8): 755-762.

7. Ando Y, Okada H, Takemura G, Suzuki K, Takada C, et al. (2018) BrainSpecific Ultrastructure of Capillary Endothelial Glycocalyx and Its Possible Contribution for Blood Brain Barrier. Sci Rep 8(1): 17523.

8. Reitsma S, Slaaf DW, Vink H, van Zandvoort MA, oude Egbrink MG (2007) The endothelial glycocalyx: composition, functions, and visualization. Pflugers Arch 454(3): 345-359.

9. Curry FE, Adamson RH (2012) Endothelial glycocalyx: permeability barrier and mechanosensor. Ann Biomed Eng 40(4): 828-839.

10.Fu BM, Tarbell JM (2013) Mechano-sensing and transduction by endothelial surface glycocalyx: composition, structure, and function. Wiley Interdiscip Rev Syst Biol Med 5(3): 381-390.

11. Harding IC, Mitra R, Mensah SA, Nersesyan A, Bal NN, et al. (2019) Endothelial barrier reinforcement relies on flow-regulated glycocalyx, a potential therapeutic target. Biorheology 56(2-3): 131-149.

12. Maroski J, Vorderwülbecke BJ, Fiedorowicz K, Da Silva Azevedo L, Siegel G, et al. (2011) Shear stress increases endothelial hyaluronan synthase 2 and hyaluronan synthesis especially in regard to an atheroprotective flow profile. Exp Physiol 96(9): 977-986.

13. Gouverneur M, Berg B, Nieuwdorp M, Stroes E, Vink H (2006) Vasculoprotective properties of the endothelial glycocalyx: effects of fluid shear stress. J Intern Med 259(4): 393-400.

14. van Haaren PM, VanBavel E, Vink H, Spaan JA (2005) Charge modification of the endothelial surface layer modulates the permeability barrier of isolated rat mesenteric small arteries. American journal of physiology. Am J Physiol Heart Circ Physiol 289(6): H2503-2507.

15. Ueda A, Shimomura M, Ikeda M, Yamaguchi R, Tanishita K (2004) Effect of glycocalyx on shear-dependent albumin uptake in endothelial cells. American journal of physiology. Am J Physiol Heart Circ Physiol 287(5): H2287-2294. 
16. Lopez Quintero SV, Cancel LM, Pierides A, Antonetti D, Spray DC, et al (2013) High glucose attenuates shear-induced changes in endothelial hydraulic conductivity by degrading the glycocalyx. PLoS One 8(11): e78954.

17. Rao G, Ding HG, Huang W, Le D, Maxhimer JB, et al. (2011) Reactive oxygen species mediate high glucose-induced heparanase- 1 production and heparan sulphate proteoglycan degradation in human and rat endothelial cells: a potential role in the pathogenesis of atherosclerosis. Diabetologia 54(6): 1527-1538.

18. Vink H, Duling BR (2000) Capillary endothelial surface layer selectively reduces plasma solute distribution volume. American journal of physiology. Am J Physiol Heart Circ Physiol 278(1): H285-289.

19. Zuurbier CJ, Demirci C, Koeman A, Vink H, Ince C (2005) Short-term hyperglycemia increases endothelial glycocalyx permeability and acutely decreases lineal density of capillaries with flowing red blood cells. J Appl Physiol 99(4): 1471-1476.

20. Dogne S, Rath G, Jouret F, Caron N, Dessy C, et al. (2016) Hyaluronidase 1 Deficiency Preserves Endothelial Function and Glycocalyx Integrity in Early Streptozotocin-Induced Diabetes. Diabetes 65(9): 2742-2753.

21. Nieuwdorp M, Mooij HL, Kroon J, Atasever B, Spaan JA, et al. (2006) Endothelial glycocalyx damage coincides with microalbuminuria in type 1 diabetes. Diabetes 55(4): 1127-1132.

22. Nieuwdorp M, van Haeften TW, Gouverneur MC, Mooij HL, van Lieshout $\mathrm{MH}$, et al. (2006) Loss of endothelial glycocalyx during acute hyperglycemia coincides with endothelial dysfunction and coagulation activation in vivo. Diabetes 55(2): 480-486.

23. Broekhuizen LN, Lemkes BA, Mooij HL, Meuwese MC, Verberne H, et al. (2010) Effect of sulodexide on endothelial glycocalyx and vascular permeability in patients with type 2 diabetes mellitus. Diabetologia 53(12): 2646-2655.
24. Lauver DA, Lucchesi BR (2006) Sulodexide: a renewed interest in this glycosaminoglycan. J Blood Med 24(3-4): 214-226.

25. Lasierra Cirujeda J, Coronel P, Aza M, Gimeno M Use of sulodexide in patients with peripheral vascular disease. J Blood Med 1: 105-115.

26. Kennett EC, Davies MJ (2007) Degradation of matrix glycosaminoglycans by peroxynitrite/peroxynitrous acid: evidence for a hydroxyl-radicallike mechanism. Free Radic Biol Med 42(8): 1278-1289.

27. Katsumura C, Sugiyama T, Nakamura K, Obayashi H, Hasegawa G, et al. (2004) Effects of advanced glycation end products on hyaluronan photolysis: a new mechanism of diabetic vitreopathy. Ophthalmic Res 36(6): 327-331.

28. Hiebert LM, Han J, Mandal AK (2014) Glycosaminoglycans, hyperglycemia, and disease. Antioxid Redox Signal 21(7): 1032-1043.

29. Dogné S, Flamion B, Caron N (2018) Endothelial Glycocalyx as a Shield Against Diabetic Vascular Complications: Involvement of Hyaluronan and Hyaluronidases. Arterioscler Thromb Vasc Biol 38(7): 1427-1439

30. Han J, Zhang F, Xie J, Linhardt RJ, Hiebert LM (2009) Changes in cultured endothelial cell glycosaminoglycans under hyperglycemic conditions and the effect of insulin and heparin. Cardiovasc Diabetol 8: 46.

31. Kutuzov N, Flyvbjerg H, Lauritzen M (2018) Contributions of the glycocalyx, endothelium, and extravascular compartment to the bloodbrain barrier. Proc Natl Acad Sci U S A 115(40): E9429-E9438.

32. Rensma SP, van Sloten TT, Launer LJ, Stehouwer CDA (2018) Cerebral small vessel disease and risk of incident stroke, dementia and depression, and all-cause mortality: A systematic review and metaanalysis. Neurosci Biobehav Rev 90: 164-173.

33. Pahakis MY, Kosky JR, Dull RO, Tarbell JM (2007) The role of endothelial glycocalyx components in mechanotransduction of fluid shear stress. Biochem Biophys Res Commun 355(1): 228-233. 